\title{
MACROPHAGE MIGRATION INHIBITORY FACTOR -173 G/C POLYMORPHISM IS NOT ASSOCIATED WITH AN INCREASED RISK OF NECROTIZING ENTEROCOLITIS IN PRETERM NEONATES IN NICU
}

\author{
G. Prencipe ${ }^{1}$, F. Piersigilli ${ }^{2}$, M.P. Ronchetti ${ }^{2}$, R. Inglese ${ }^{3}$, F. De Benedetti ${ }^{4}$, C. Auriti ${ }^{2}$ \\ ${ }^{1}$ Rheumatic Research Laboratory, ${ }^{2}$ Neonatology, ${ }^{3}$ Clinical Chemistry Laboratory, ${ }^{4}$ Pediatrics, Bambino \\ Gesù Children's Hospital, Rome, Italy
}

Background: Necrotizing Enterocolitis (NEC) is a leading cause of morbidity and mortality among preterm neonates. To identify subsets of neonates at high risk of NEC is a challenge. Local pro-inflammatory response of the host could be of relevance in the still unclear pathogenesis of NEC. Macrophage migration inhibitory factor (MIF) is a pro-inflammatory cytokine, implicated in the pathophysiology of inflammatory bowel diseases, such as chronic colitis and gastric inflammation. The MIF promoter contains a functionally relevant single nucleotide polymorphism (SNP) G/C at position -173 . The MIF $-173 \mathrm{C}$ allele is associated with higher MIF expression in vitro.

Aims: The aim of the study was to investigate whether the G/C polymorphism at -173 of the MIF promoter could be a risk factor for NEC and mortality during NEC, in preterm neonates.

Material and methods: In a retrospective study we enrolled 107, GA $\leq 32$ weeks, neonates admitted to NICU. Among them 41 were affected by NEC (Bell's stages I-II and III) and 66 were controls. MIF -173 genotyping was carried out by PCR and DHPLC in all enrolled patients.

Results: The frequency of the $-173 \mathrm{G} / \mathrm{C}$ polymorphism and the distribution of $-173 \mathrm{MIF}$ genotype were similar in neonates with NEC compared to controls. We did not observe any association between the $173 \mathrm{G} / \mathrm{C}$ polymorphism and mortality.

Conclusions: The G/C polymorphism at -173 of the MIF promoter does not appear of relevance to identify subsets of neonates at high risk of NEC and to predict mortality associated to NEC, in preterm neonates in NICU. 